\title{
QCD saturation in the dipole sector with correlations
}

\author{
Romuald A. Janik* \\ Institute of Physics \\ Jagellonian University \\ ul. Reymonta 4 \\ 30-059 Krakow \\ Poland
}

November 9, 2018

\begin{abstract}
In this paper we study the description of saturation in Balitsky, Jalilian-Marian, Iancu, McLerran, Weigert, Leonidov and Kovner (BJIMWLK) picture when restricted to observables made up only from dipole operators. We derive a functional form of the evolution equation for the dipole probability distribution and find a one-parameter family of exact solutions to the dipole evolution equations.
\end{abstract}

\section{Introduction}

One of the outstanding issues in perturbative QCD is the understanding of parton saturation. Standard evolution equations like BFKL [1] lead to rising cross sections (or structure functions) which violate the unitarity bound. These are modified, when one takes into account recombination effects, into an infinite set of coupled nonlinear equations for correlation functions of

*e-mail: ufrjanik@if.uj.edu.pl 
Wilson line operators [2]. This set of equations is expected to be equivalent to the Jalilian-Marian, Iancu, McLerran, Weigert, Leonidov and Kovner (JIMWLK) functional equations [3], which can be recast in the form of Fokker-Planck equations for the (functional) probability distribution of Wilson lines $Z_{Y}\left[\left\{U_{x}\right\}\right]$. The probability distribution $Z_{Y}\left[\left\{U_{x}\right\}\right]$ encodes expectation values of observables made up of the longitudinal Wilson lines $U_{x}$, where $x$ is a coordinate in the transverse space:

$$
\left\langle f\left[\left\{U_{x}\right\}\right]\right\rangle \equiv \int \mathcal{D} U_{x} f\left[\left\{U_{x}\right\}\right] Z_{Y}\left[\left\{U_{x}\right\}\right]
$$

The full knowledge of $Z_{Y}\left[\left\{U_{x}\right\}\right]$ is of course nonperturbative, but the evolution in rapidity is governed by the JIMWLK functional equation:

$$
\frac{\partial}{\partial Y} Z_{Y}\left[\left\{U_{x}\right\}\right]=\alpha_{s} \frac{1}{2} \int d^{2} x d^{2} y\left(i \nabla_{U_{x}}^{a} \hat{\chi}_{x y}^{a b} i \nabla_{U_{y}}^{b}\right) Z_{Y}\left[\left\{U_{x}\right\}\right]
$$

where the derivatives $\nabla_{U_{x}}^{a}$ are covariant functional derivatives w.r.t. the unitary matrices while the kernel $\hat{\chi}_{x y}^{a b}$ is given by the following expression:

$$
\hat{\chi}_{x y}^{a b}=\frac{1}{\pi^{2}} \int d^{2} z\left(\frac{(x-z)_{i}}{(x-z)^{2}}\left[1-U_{x}^{-1} U_{z}\right]^{a c}\right)\left(\frac{(z-y)_{i}}{(z-y)^{2}}\left[1-U_{z}^{-1} U_{y}\right]^{c b}\right)
$$

See 44 and [5] for further details. Despite the apparent compactness of the above equation which summarizes the whole nonlinear infinite set of evolution equations for correlation functions it has proved itself to be difficult to analyze. Apart from some general properties not much is explicitly known about the details of saturation/unitarization in this picture.

It is interesting, therefore, to study simplified versions. One noteworthy example is the Balitsky-Kovchegov (BK) equation [2, 6] obtained when correlation functions of traces of products Wilson lines factorize into products of expectation values. This leads to a nonlinear equation for the dipole density

$$
\frac{\partial}{\partial Y}\left\langle\mathcal{S}_{01}\right\rangle=\frac{g^{2}}{8 \pi^{3}} \int d^{2} x_{2} \frac{x_{01}^{2}}{x_{02}^{2} x_{21}^{2}}\left[\left\langle\mathcal{S}_{02}\right\rangle\left\langle\mathcal{S}_{21}\right\rangle-N_{c}\left\langle\mathcal{S}_{01}\right\rangle\right]
$$

where the $\mathcal{S}_{i j}$ are (the S-matrix versions of) the dipole operators

$$
\mathcal{S}_{i j}=\operatorname{tr} U_{x_{i}} U_{x_{j}}^{\dagger}
$$

The Balitsky-Kovchegov equation was studied both numerically and analytically [7] and boasts universal properties of geometrical scaling [8] (appearing here as universal traveling wave solutions [9]). 
In order to better understand the full hierarchy of JIMWLK equations, it is of particular interest to find some intermediate approximations which are not so drastic as BK, yet remain simpler than the full JIMWLK hierarchy. One approach might be to restrict oneself just to observables made up from dipole operators, as these have a very clear intuitive physical meaning, yet to refrain from making any other simplifying assumptions. The generalization with respect to BK means that one has to allow for nontrivial correlations between dipoles i.e. allowing for $\left\langle\mathcal{S}_{01} \mathcal{S}_{23}\right\rangle \neq\left\langle\mathcal{S}_{01}\right\rangle\left\langle\mathcal{S}_{23}\right\rangle$ etc. In 10] we studied the first step beyond BK with nontrivial 2-dipole correlations. Other approaches going beyond BK include [11, 12, 13.

The aim of this paper is to derive an analogue of (2) restricted to probability distributions made up of dipole operators only, and to find a oneparameter class of exact solutions of these dipole evolution equations with some specific multi-dipole correlations.

The comparison of the dipole evolution hamiltonian to the full JIMWLK one may be a first step in understanding the role of higher multipole operators in perturbative unitarization/saturation processes.

The plan of this paper is as follows. In section 2 we derive the evolution hamiltonian for the JIMWLK hierarchy restricted to dipole operators, then in section 3 we discuss some of its properties. In section 4 we construct a one-parameter solution of the dipole evolution equations and we close the paper with a summary and discussion.

\section{The JIMWLK hierarchy restricted to dipoles}

In order to calculate the evolution of an observable of the form $\left\langle\mathcal{S}_{01} \mathcal{S}_{23} \ldots \mathcal{S}_{n-1 n}\right\rangle$ it is most convenient to use Balitsky's approach where the evolution with rapidity is obtained using contractions between pairs of the unitary matrices $U_{x}$ 's appearing in the dipole operators and virtual corrections for each $U_{x}$ according to eqs. (119), (120) in [2]. One can verify that contractions between $U_{x}$ 's belonging to different dipoles always give rise to multipoint traces, so if one wants to keep only terms involving dipoles one performs contractions only within each dipole and therefore each dipole operator evolves independently through the operator form of the BK kernel:

$$
\frac{\partial}{\partial Y} f\left[\mathcal{S}_{x y}\right]=\frac{g^{2}}{8 \pi^{3}} \int d^{2} x d^{2} y d^{2} z\left(\frac{\delta}{\delta S_{x y}} f\left[\mathcal{S}_{x y}\right]\right) K_{x z y}\left(\mathcal{S}_{x z} \mathcal{S}_{z y}-N_{c} \mathcal{S}_{x y}\right)
$$


Introducing the dipole probability distribution $Z_{Y}\left[\left\{\mathcal{S}_{x y}\right\}\right]$ which encodes expectation values through

$$
\left\langle f\left[\left\{\mathcal{S}_{x y}\right\}\right]\right\rangle \equiv \int \mathcal{D} \mathcal{S}_{x y} f\left[\left\{\mathcal{S}_{x y}\right\}\right] Z_{Y}\left[\left\{\mathcal{S}_{x y}\right\}\right]
$$

we can rewrite the previous equation as

$$
\begin{aligned}
\frac{\partial}{\partial Y}\left\langle f\left[\mathcal{S}_{x y}\right]\right\rangle= & \frac{g^{2}}{8 \pi^{3}} \int \mathcal{D} \mathcal{S}_{x y} \int d^{2} x d^{2} y d^{2} z\left(\frac{\delta}{\delta S_{x y}} f\left[\mathcal{S}_{x y}\right]\right) . \\
& \cdot K_{x z y}\left(\mathcal{S}_{x z} \mathcal{S}_{z y}-N_{c} \mathcal{S}_{x y}\right] Z_{Y}\left[\left\{\mathcal{S}_{x y}\right\}\right]
\end{aligned}
$$

We will now recast the right hand side as

$$
\int \mathcal{D} \mathcal{S}_{x y} f\left[\mathcal{S}_{x y}\right] \mathcal{H} Z_{Y}\left[\left\{\mathcal{S}_{x y}\right\}\right]
$$

Then the evolution equation for the dipole probability distribution will have the form

$$
\frac{\partial}{\partial Y} Z_{Y}\left[\left\{\mathcal{S}_{x y}\right\}\right]=\mathcal{H} Z_{Y}\left[\left\{\mathcal{S}_{x y}\right\}\right]
$$

If we perform a functional integration by parts in (8) and assume the vanishing of boundary terms we obtain an explicit and quite simple form of the evolution hamiltonian $\mathcal{H}$ :

$$
\frac{\partial}{\partial Y} Z_{Y}\left[\left\{\mathcal{S}_{x y}\right\}\right]=\frac{-g^{2}}{8 \pi^{3}} \int d^{2} x d^{2} y d^{2} z \frac{\delta}{\delta S_{x y}}\left\{K_{x z y}\left(\mathcal{S}_{x z} \mathcal{S}_{z y}-N_{c} \mathcal{S}_{x y}\right)\right\} Z_{Y}\left[\left\{\mathcal{S}_{x y}\right\}\right]
$$

\section{Some remarks on the dipole hamiltonian}

As an example of how the functional evolution equation (11) works in practice let us quickly rederive the equation for 2-dipole correlations obtained in [10]. Let us start from the identity

$$
\frac{\partial}{\partial Y}\left\langle\mathcal{S}_{01} \mathcal{S}_{23}\right\rangle=\int \mathcal{D} \mathcal{S}_{x y} \mathcal{S}_{01} \mathcal{S}_{23} \frac{\partial}{\partial Y} Z_{Y}\left[\left\{\mathcal{S}_{x y}\right\}\right]
$$

Now we use (11) and perform integration by parts to get

$$
\frac{g^{2}}{8 \pi^{3}} \int \mathcal{D} \mathcal{S}_{x y} \int d^{2} x d^{2} y d^{2} z \frac{\delta}{\delta S_{x y}}\left(\mathcal{S}_{01} \mathcal{S}_{23}\right)\left\{K_{x z y}\left(\mathcal{S}_{x z} \mathcal{S}_{z y}-N_{c} \mathcal{S}_{x y}\right)\right\} Z_{Y}\left[\left\{\mathcal{S}_{x y}\right\}\right]
$$


After performing the functional differentiation and using the definition of $Z_{Y}\left[\left\{\mathcal{S}_{x y}\right\}\right]$ (77) we get the equation obtained in [10]:

$$
\begin{aligned}
\frac{\partial}{\partial Y}\left\langle\mathcal{S}_{02} \mathcal{S}_{2^{\prime} 1}\right\rangle= & \frac{g^{2}}{8 \pi^{3}} \int d^{2} x_{3}\left[\left\langle\mathcal{S}_{03} \mathcal{S}_{32} \mathcal{S}_{2^{\prime} 1}\right\rangle-N_{c}\left\langle\mathcal{S}_{02} \mathcal{S}_{2^{\prime} 1}\right\rangle\right] K_{032}+ \\
& +\left[\left\langle\mathcal{S}_{2^{\prime} 3} \mathcal{S}_{31} \mathcal{S}_{02}\right\rangle-N_{c}\left\langle\mathcal{S}_{02} \mathcal{S}_{2^{\prime} 1}\right\rangle\right] K_{132^{\prime}}
\end{aligned}
$$

Let us now contrast some properties of the dipole evolution equation (11) with the full JIMWLK one (2). The latter one is second order in functional derivatives and, as shown by Weigert, the hamiltonian is positive definite, with a unique attractive fixed point $\left(Z_{Y}\left[\left\{U_{x}\right\}\right]=1\right)$. On the other hand the dipole equation is first order and as such does not seem to have such positivity and uniqueness properties. Even more so, from the form of (11) one would expect that an infinite set of zero-modes would exist. This would lead to many probability distributions determined by

$$
\mathcal{H} Z_{Y}\left[\left\{\mathcal{S}_{x y}\right\}\right]=0
$$

which are invariant under evolution.

We cannot a-priori rule this out but let us show on a simple toy model how one can nevertheless escape this conclusion. Assume the following simple evolution equation for a dipole operator

$$
\frac{\partial}{\partial Y} s=s-s^{2}
$$

which exhibits saturation behaviour quite similar to BK. This leads to the hamiltonian for the probability distribution $Z_{Y}[s]$ :

$$
\frac{\partial}{\partial Y} Z_{Y}[s]=\partial_{s}\left[\left(s^{2}-s\right) Z_{Y}[s]\right]
$$

One can now find explicitly the dangerous stationary solutions:

$$
Z_{Y}[s]=\frac{c}{s(1-s)}
$$

However these solutions are nonphysical as they are nonnormalizable due to the singularities at $s=0,1$, and therefore cannot represent any probability distribution. It would be very interesting (but rather difficult) to carry out a similar analysis for the real dipole equation (11). 


\section{A family of factorized solutions to the dipole hierarchy of evolution equations}

It is interesting to look for exact solutions of the full set of dipole evolution equations. It turns out to be easier to find a solution of the type considered here using directly a set of evolution equations for the moments of dipole operators rather than finding first the probability distribution $Z_{Y}\left[\left\{\mathcal{S}_{x y}\right\}\right]$.

The JIMWLK-Balitsky hierarchy of evolution equations, when restricted to dipole operators has the following form:

$$
\frac{\partial}{\partial Y} \mathcal{S}_{1} \mathcal{S}_{2} \ldots \mathcal{S}_{n}=\sum_{i=1}^{n} \mathcal{S}_{1} \ldots \mathcal{S}_{i-1}\left(\mathcal{H} \mathcal{S}_{i}\right) \mathcal{S}_{i+1} \ldots \mathcal{S}_{n}
$$

In terms of dipole density operators $\mathcal{N}_{i}$ related to the $\mathcal{S}_{i}$ through

$$
\mathcal{S}_{i}=N_{c}\left(1-\mathcal{N}_{i}\right)
$$

the hamiltonian acts as

$$
\mathcal{H} \mathcal{N}_{i} \equiv \mathcal{H} \mathcal{N}_{i i^{\prime}}=\frac{g^{2} N_{c}}{8 \pi^{3}} \int d^{2} x K_{i x i^{\prime}}\left[\mathcal{N}_{i x}+\mathcal{N}_{x i^{\prime}}-\mathcal{N}_{i i^{\prime}}-\mathcal{N}_{i x} \mathcal{N}_{x i^{\prime}}\right]
$$

Let us look for solutions to this set of coupled equations in a factorized form:

$$
\left\langle\mathcal{N}_{1} \mathcal{N}_{2} \ldots \mathcal{N}_{n}\right\rangle=c_{n}\left\langle\mathcal{N}_{1}\right\rangle\left\langle\mathcal{N}_{2}\right\rangle \ldots\left\langle\mathcal{N}_{n}\right\rangle \equiv c_{n} N_{1} N_{2} \ldots N_{n}
$$

with $c_{n}$ some coefficients $\left(c_{1} \equiv 1\right)$. Note that $\langle\ldots\rangle$ is the ordinary expectation value and not just the connected part. If all $c_{n}$ would be equal to one, this would imply no nontrivial correlations and would be equivalent to the standard BK equation.

Let us take the expectation value in the evolution equation (19):

$$
\begin{gathered}
\frac{\partial}{\partial Y}\left\langle\left(1-\mathcal{N}_{1}\right) \ldots\left(1-\mathcal{N}_{n}\right)\right\rangle=-\sum_{i=1}^{n}\left\langle\left(1-\mathcal{N}_{1}\right) \ldots\left(1-\mathcal{N}_{i-1}\right)\left(\mathcal{H} \mathcal{N}_{i}\right)\left(1-\mathcal{N}_{i+1}\right)\right. \\
\left.\ldots\left(1-\mathcal{N}_{n}\right)\right\rangle
\end{gathered}
$$

We may now use first the assumption of factorization (22) in the left hand side of (23). Thus the $Y$-derivative acts on products of single dipole densities $N_{i}=\left\langle\mathcal{N}_{i}\right\rangle$. Then for each $N_{i}$ we use the evolution operator

$$
\frac{\partial}{\partial Y} N_{1}=\frac{g^{2} N_{c}}{8 \pi^{3}} \int d^{2} x K_{i x i^{\prime}}\left[N_{i x}+N_{x i^{\prime}}-N_{i i^{\prime}}-c_{2} N_{i x} N_{x i^{\prime}}\right]
$$


Note the appearance of $c_{2}$ w.r.t. (21) as we are taking expectation values. We can now insert the operator form of (21) into the right hand side of (23) and then perform the averaging using the factorization ansatz (22). Comparing terms we see that all terms match if the following holds:

$$
c_{i+1}=c_{2} c_{i}
$$

which has a simple solution ${ }^{1}$

$$
c_{n}=c_{2}^{n-1}
$$

Therefore all multidipole correlation functions reduce to one-dipole densities but with an enhancement factor:

$$
\left\langle\mathcal{N}_{1} \mathcal{N}_{2} \ldots \mathcal{N}_{n}\right\rangle=c_{2}^{n-1} N_{1} N_{2} \ldots N_{n}
$$

And all the one dipole densities $N_{i}$ satisfy a modified BK equation

$$
\frac{\partial}{\partial Y} N_{i i^{\prime}}=\frac{g^{2} N_{c}}{8 \pi^{3}} \int d^{2} x K_{i x i^{\prime}}\left[N_{i x}+N_{x i^{\prime}}-N_{i i^{\prime}}-c_{2} N_{i x} N_{x i^{\prime}}\right]
$$

with $c_{2}$ being an a-priori arbitrary parameter. However in order to have a solution which saturates at $N_{i} \leq 1, c_{2}$ has to be greater or equal to 1 . Indeed let us note that (27) with $N_{i}=1 / c_{2}$ is a fixed point of the dipole evolution equations. It would be interesting to recast this solution in the language of probability distributions $Z_{Y}\left[\left\{\mathcal{S}_{x y}\right\}\right]$ and analyze its normalizability properties.

We note that the same modified equation was proposed in [1] to account for effects of correlations in nuclei on saturation. It is interesting to note that the same equation arises from an exact solution of the dipole evolution hierarchy.

Let us note how does the factorized solution of [10] fit into the above general framework. That solution was obtained assuming that the dipole evolution equations close at the level of two equations for $\left\langle\mathcal{N}_{1}\right\rangle$ and $\left\langle\mathcal{N}_{1} \mathcal{N}_{2}\right\rangle$ i.e. that there are no nontrivial 3-dipole correlations. In order to recover that solution in our framework we have to analyze the connected correlation functions

$$
\left\langle\mathcal{N}_{1} \mathcal{N}_{2} \ldots \mathcal{N}_{n}\right\rangle_{c}=d_{n} N_{1} N_{2} \ldots N_{n}
$$

\footnotetext{
${ }^{1}$ After these results were obtained M. Lublinsky informed me that the same factorized solution appeared in a framework of generalized BK in [1].
} 
The coefficients $d_{n}$ are related to the $c_{n}$ 's through

$$
\begin{aligned}
& c_{2}=c_{1}^{2}+d_{2} \\
& c_{3}=c_{1}^{3}+3 c_{1} d_{2}+d_{3} \\
& c_{4}=c_{1}^{4}+6 d_{2} c_{1}^{2}+3 d_{2}^{2}+4 c_{1} d_{3}+d_{4}
\end{aligned}
$$

etc. The requirement that connected 3-dipole correlations vanish leads to the equation $d_{3}=c_{2}^{2}-1-3\left(c_{2}-1\right)=0$ which fixes $c_{2}$ to be either 1 (no correlations at all) or $c_{2}=2$ (the solution found in [10]). We note that from the general formula (26) that solution (for $\left\langle\mathcal{N}_{1}\right\rangle$ and $\left\langle\mathcal{N}_{1} \mathcal{N}_{2}\right\rangle$ ) can be extended to a solution of the full dipole hierarchy but with some nonvanishing higher-dipole correlations (like $d_{4} \neq 0$ ).

\section{Summary}

In this paper we studied the JIMWLK evolution equations in the approxmation of keeping only dipole-like color contractions and neglecting all higher multipole operators. In this approximation we have derived an equation for the evolution of the dipole probability distribution. No further assumptions like neglecting correlations were made. The hamiltonian turned out to be first order in functional derivatives in contrast to the full JIMWLK evolution. It would be interesting to study further properties of this hamiltonian.

In the second part of the paper we derived a one-parameter family of exact solutions to the dipole evolution hierarchy. They are characterized by an enhancement (or depletion) factor in multidipole correlation functions and the single dipole densities are governed by a modified BK equation.

It seems that it would be very interesting to study further the properties of saturation when restricted to the dipole sector since this is a much simpler system than the full JIMWLK one. In particular one could explore the probabilisitic interpretation of the dipole evolution equations (11), the structure of (attractive) fixed points and the question whether multipole operators present in the full JIMWLK framework lead to qualitative or just quantitative changes in the physics of saturation.

Acknowledgements. I would like to thank Edmond Iancu, Michael Lublinsky and Robi Peschanski for discussions and comments. This work was supported in part by KBN grants 2P03B09622 (2002-2004), 2P03B08225 (20032006) and 1P03B02427 (2004-2007). 


\section{References}

[1] L. N. Lipatov, Sov. J. Nucl. Phys. 23, 338 (1976); E. A. Kuraev, L. N. Lipatov, and V. S. Fadin, Sov. Phys. JETP 45, 199 (1977); I. I. Balitsky and L. N. Lipatov, Sov. J. Nucl. Phys. 28, 822 (1978).

[2] I. Balitsky, Nucl. Phys. B463 (1996) 99.

[3] J.Jalilian-Marian,A. Kovner, A. Leonidov and H. Weigert, Nucl. Phys. B504 (1997) 415; Phys. Rev. D59 (1999) 014014. H. Weigert, Nucl. Phys. A703 (2002) 823. E.Iancu, A. Leonidov and L. McLerran, Nucl.Phys. A692 (2001) 583; Phys.Lett. B510 (2001) 133.

[4] H. Weigert, Nucl. Phys. A 703 (2002) 823 arXiv:hep-ph/0004044.

[5] J. P. Blaizot, E. Iancu and H. Weigert, Nucl. Phys. A 713, 441 (2003) arXiv:hep-ph/0206279.

[6] Y. V. Kovchegov, Phys. Rev. D60, 034008 (1999); Phys. Rev. D61, 074018 (2000).

[7] See e.g. K. Golec-Biernat and A. M. Stasto, Nucl. Phys. B 668 (2003) 345 arXiv:hep-ph/0306279. A. H. Mueller and D. N. Triantafyllopoulos, Nucl. Phys. B 640 (2002) 331 arXiv:hep-ph/0205167. E. Iancu, K. Itakura and L. McLerran, Nucl. Phys. A 708, 327 (2002) arXiv:hep-ph/0203137 . J. Bartels, E. Gotsman, E. Levin, M. Lublinsky and U. Maor, Phys. Rev. D 68 (2003) 054008 arXiv:hep-ph/0304166.

[8] A. M. Staśto, K. Golec-Biernat, and J. Kwiecinski, Phys. Rev. Lett. 86, 596 (2001), hep-ph/0007192.

[9] S. Munier and R. Peschanski, Phys. Rev. Lett. 91, 232001 (2003); Phys. Rev. D D69, 034008 (2004); hep-ph/0401215.

[10] R. A. Janik and R. Peschanski, arXiv:hep-ph/0407007.

[11] E. Levin and M. Lublinsky, Nucl. Phys. A 730 (2004) 191 arXiv:hep-ph/0308279.

[12] E. Iancu and A. H. Mueller, Nucl. Phys. A 730, 494 (2004) arXiv:hep-ph/0309276. 
[13] A. H. Mueller and A. I. Shoshi, Nucl. Phys. B 692 (2004) 175 arXiv:hep-ph/0402193. 